\title{
Anti-TNF- $\alpha$-Therapie der Psoriasis mit Infliximab oder Etanercept. Klinischer, histologischer und immunhistochemischer Verlauf ${ }^{1}$
}

\author{
Anti-TNF- $\alpha$-Therapy of Psoriasis with Infliximab or Etanercept. Clinical, Histological and Immunohistochemical \\ Course
}

Autoren

Institut

\section{Angsten, R. E. Schopf}

Universitäts-Hautklinik, Johannes Gutenberg-Universität Mainz

\section{Bibliografie}

DOI $10.1055 / \mathrm{s}-2007-966548$

Online-Publikation: 23. 7. 2007

Akt Dermatol 2007; 33:

310-316 @ Georg Thieme Verlag KG Stuttgart · New York ISSN 0340-2541

Korrespondenzadresse Dr. Marina Angsten

Kaiser-Wilhelm-Ring 31-33

55118 Mainz

marina.angsten@

onlinehome.de

\section{Zusammenfassung \\ $\nabla$}

TNF- $\alpha$ spielt eine zentrale Rolle in der Pathogenese der Psoriasis. In vorangehenden Studien verbesserten sich psoriatische Hautläsionen unter anti-TNF- $\alpha$-Therapie mit Etanercept oder Infliximab. Beide Medikamente wirken über einen unterschiedlichen, noch nicht vollständig geklärten Mechanismus und differieren bezüglich des Wirkspektrums. Diese Arbeit untersucht und vergleicht bei 12 Patienten die Effekte der Kurzzeittherapien mit Etanercept (6 Patienten) oder Infliximab (6 Patienten) auf die verschiedenen pathohistologischen bzw. klinischen Befunde der Psoriasis. Zur klinischen Betrachtung dienen PASI und Juckreiz. Hautbiopsien und deren HE- bzw. immunhistochemische Färbungen ermöglichen die Beurteilung der histologischen Befunde (Epidermisdicke, Entzündungsreaktion, Proliferations- und Differenzierungsstörung der Keratinozyten). Die deskriptive Analyse erfolgt mittels Median und Quartilen (1. und 3. Quartil). Etanercept bewirkt eine Reduktion des PASI um 8,2 $(14,7 ; 6,7 ; p=0,031)$. Der Juckreiz nach Behandlung mit Etanercept ist vermindert oder unverändert, die Epidermisdicke hat sich normalisiert

\section{Einleitung}

$\nabla$

TNF- $\alpha$ spielt eine zentrale Rolle in der Pathogenese der Psoriasis [1]. Das proinflammatorische Zytokin fördert die Immunantwort, die Keratinozytenproliferation und die Expression von Adhäsionsmolekülen, welche für die T-Zell-Migration notwendig sind [2]. TNF- $\alpha$ kommt als inaktives Monomer und aktives Trimer vor. Neben einer löslichen Form existiert auch eine membranstän-

\footnotetext{
${ }^{1}$ Die Arbeit bezieht sich auf Ergebnisse der gleichnamigen Dissertation von Frau Marina Angsten.
}

$(p=0,031)$. Der gleiche Trend zeichnet sich hinsichtlich der Proliferations- und Differenzierungsstörung ab. Die epidermalen und dermalen Entzündungsparameter haben sich insgesamt betrachtet jedoch nicht verringert. Infliximab bewirkt einen Abfall des PASI um 18,5 (23,6; 12,9; $\mathrm{p}=0,031$ ). Auch Juckreiz und Epidermisdicke haben sich nach Therapie verbessert (jeweils $\mathrm{p}=0,031$ ). Die Proliferations- und Differenzierungsstörung der Keratinozyten normalisiert sich ebenfalls (für Ki-67 und Involucrin, jeweils $\mathrm{p}=0,031$ ). Im Gegensatz zur Etanerceptgruppe spricht in der Infliximabgruppe auch das entzündliche Infiltrat für eine Abheilung der psoriatischen Plaques (bei CD45RO+-T-Zellen, $\mathrm{p}=0,031$ ). Die Infliximabgruppe zeichnet sich bei allen klinischen und histologischen Befunden durch eine stärkere Verbesserung des Hautstatus aus. Seitens der Entzündungsreaktion zeigt sich hinsichtlich der CD3+-T-Zellen ein Rückgang $(p=0,032)$. Die dargelegten Befunde sind mit den unterschiedlichen Wirkmechanismen von Infliximab und Etanercept vereinbar und spiegeln die Schlüsselposition von TNF- $\alpha$ bezüglich der Psoriasis wider. 


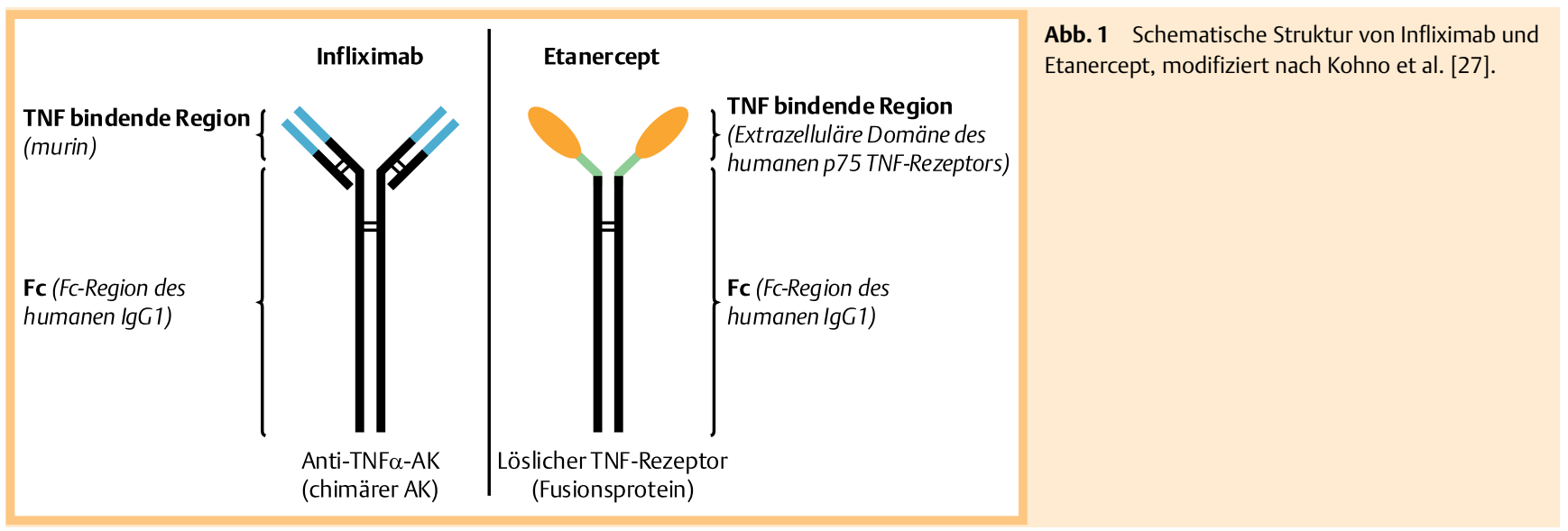

unterscheiden sich chemisch und im Wirkmechanismus, der noch nicht vollständig geklärt ist, und differieren bezüglich des Wirkspektrums. Etanercept ist ein vollständig humanes Fusionsprotein, bestehend aus dem menschlichen p75TNFR, verbunden mit der Fc-Region des humanen IgG1 [8]. Es ahmt die Wirkungsweise der natürlich vorkommenden löslichen TNF- $\alpha$-Rezeptoren nach, jedoch mit einer höheren Affinität zu TNF- $\alpha[9,10]$.

Bei Infliximab handelt es sich um einen chimären human-murinen AK, der spezifisch gegen menschliches TNF- $\alpha$ gerichtet ist [11]. Klinische Untersuchungen belegen im Gegensatz zu Etanercept die Wirksamkeit von Infliximab bei M. Crohn [12,13]. Die Infliximabtherapie hat bezüglich granulomatöser Reaktionen (z.B. Tbc) ein höheres Risiko und zeigt ein früheres Auftreten dieser Granulome als die Etanercepttherapie [14-17].

Das Ziel der Untersuchung besteht in der Darstellung der Wirkungen der TNF- $\alpha$-Blocker Etanercept bzw. Infliximab auf die verschiedenen pathologischen Faktoren der Psoriasis vulgaris. Dazu erfolgt die klinische, histologische und immunhistochemische Untersuchung der Haut von Psoriatikern vor Therapie und nach Kurzzeitbehandlung mit Etanercept oder Infliximab.

\section{Patienten, Material und Methoden \\ $\nabla$}

Patientenkollektiv, Therapie und Probeentnahme

Untersucht wurden 12 männliche Patienten der Poliklinik (Hautklinik der Universitätsklinik Mainz) im Alter von 32-59 Jahren mit einer mittelschweren bis schweren Psoriasis vulgaris (Psoriasis Area and Severity Index (PASI) $\geq 12$ ). 6 Patienten erhalten das Medikament Infliximab, die anderen 6 Patienten Etanercept. Den Patienten der Infliximabgruppe wird eine Infusion mit Remicade ${ }^{\circledR}$ in Woche 0, 2 und 6 (5 mg/kg Körpergewicht) verabreicht. Enbrel ${ }^{\circledR}$ wird den Patienten der Etanerceptgruppe 2-mal wöchentlich subkutan gespritzt (2-mal $25 \mathrm{mg} /$ Woche subku$\tan$ ). Hautproben wurden vor Therapie (Woche 0 ) aus unbefallener und befallener Haut und während der Therapie aus befallener Haut (Infliximabgruppe nach Woche 10, Etanerceptgruppe nach Woche 12) entnommen.

\section{Erhebung der Zielgrößen}

Zum Zeitpunkt der Probeentnahmen (PEs) werden klinische Parameter erhoben (PASI [18] und Juckreiz). Die Schwere des Juckreizes wird entsprechend den Angaben der Patienten festgehalten (0: kein, 1: leicht, 2: mittelschwer, 3: schwer).

Aus den PEs werden Paraffinschnitte hergestellt. Diese werden mit Hämatoxylin-Eosin (HE) gefärbt. Anhand dieser Schnitte er- folgt die Ausmessung der Epidermisdicke. Außerdem werden immunhistochemische Färbungen mit 8 verschiedenen Antikörpern (s. u.) nach der Labelled Streptavidin-Biotin- (LSAB-)Methode durchgeführt.

\section{Entzündungsmarker}

Es werden Primärantikörper gegen folgende Antigene auf Entzündungszellen verwendet: CD3 (Pan-T-Zellmarker), CD4 (T-Helfer-Zellen), CD8 (Zytotoxische T-Zellen) und CD45RO (Memory-T-Zellen). Die Auswertung der Entzündungsmarker erfolgt semiquantitativ (Zahl von 0-4; 0: keine Zellen angefärbt , 4: viele Zellen angefärbt).

\section{Proliferations- und Differenzierungsmarker}

Der AK (Antikörper) gegen Ki-67 ist ein Marker für den gesteigerten Zellzyklus der Psoriasis. Es wird die Anzahl der angefärbten Zellkerne ausgezählt. CK16 (Zytokeratin 16) charakterisiert den hyperproliferativen Zustand der Epidermis, es wird suprabasal exprimiert [19]. Involucrin stellt einen Marker der terminalen Differenzierung der Keratinozyten dar. In gesunder Haut befindet sich Involucrin im oberen Drittel der Epidermis [20,21], in psoriatisch veränderter Haut auch in tieferen Schichten der Epidermis [22]. Der Anti-Filaggrin-AK ist ein Marker für die Keratinisierung und somit für die Differenzierung der Epidermis. In gesunder Haut erscheint Filaggrin in Stratum corneum und Stratum granulosum, bei Psoriasis zeigt es eine verminderte Ausprägung [21,20].

\section{Deskription und Statistik}

Die deskriptive Analyse der erhobenen Parameter erfolgt jeweils getrennt nach Patientenkollektiv mittels Median, Quartilen (1. und 3. Quartil), Minimum und Maximum. Für die ordinalen Zielgrößen Entzündungsmarker und Juckreiz werden zusätzlich die absoluten Häufigkeiten (Fallzahlen) und prozentualen Häufigkeiten ermittelt. Die statistische Auswertung erfolgt mittels Vorzeichentest (intraindividuelle Differenzen zwischen Messwerten vor und nach Therapie) und unverbundenem Wilcoxontest (Mann-Whitney-U-Test, Etanercept- vs. Infliximabgruppe). Nach Eingabe aller Daten in OpenOffice Calc (1.1.3) werden diese in SPSS (11.5) überführt und statistisch ausgewertet. Die Erstellung der Diagramme erfolgt mittels OpenOffice Calc und SPSS. Alle statistischen Tests werden im Sinne einer explorativen Datenanalyse verwendet. Eine Korrektur der p-Werte wird nicht durchgeführt. P-Werte $\leq 0,05$ werden als statistisch auffällig, p-Werte $\leq 0,10$ als statistisch grenzwertig angesehen. 


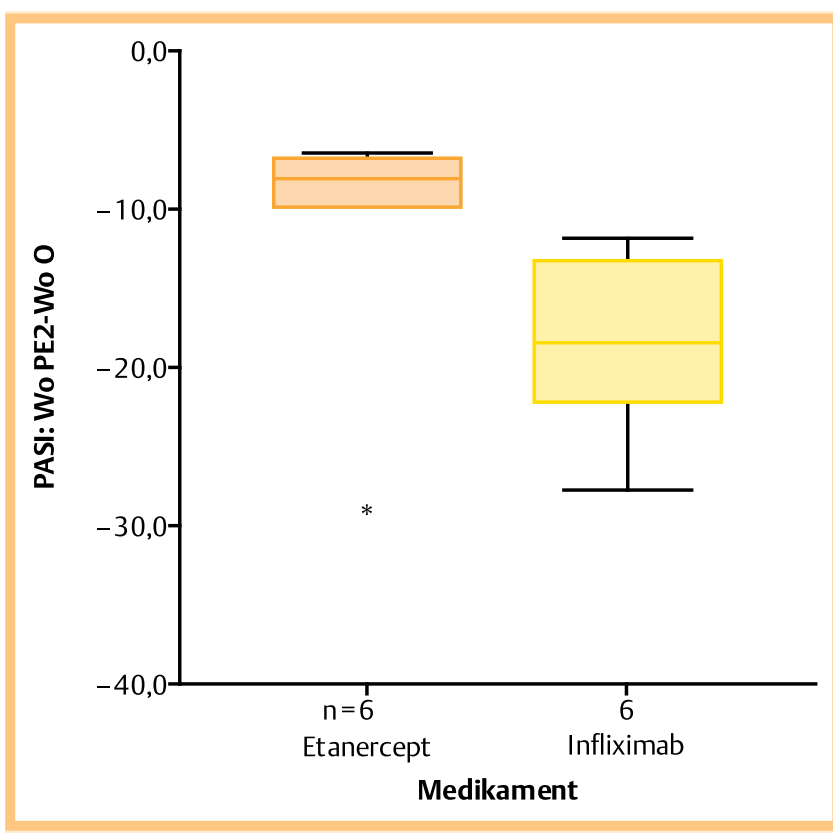

Abb. 2 PASI: Differenz des Hautbefundes vor und nach Therapie; Infliximab und Etanercept: $p$-Wert Vorzeichentest $<0,05(p=0,031)$.

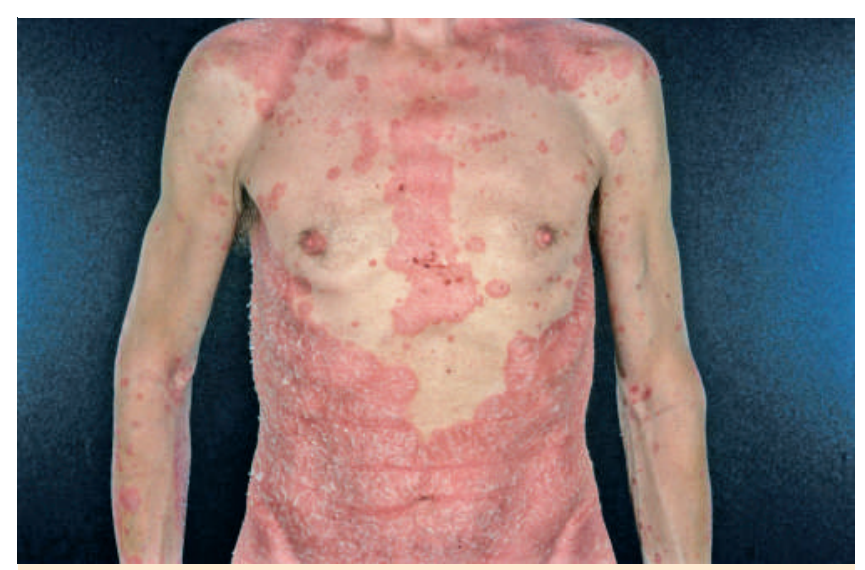

Abb. 3 Etanerceptpatient vor Therapie.

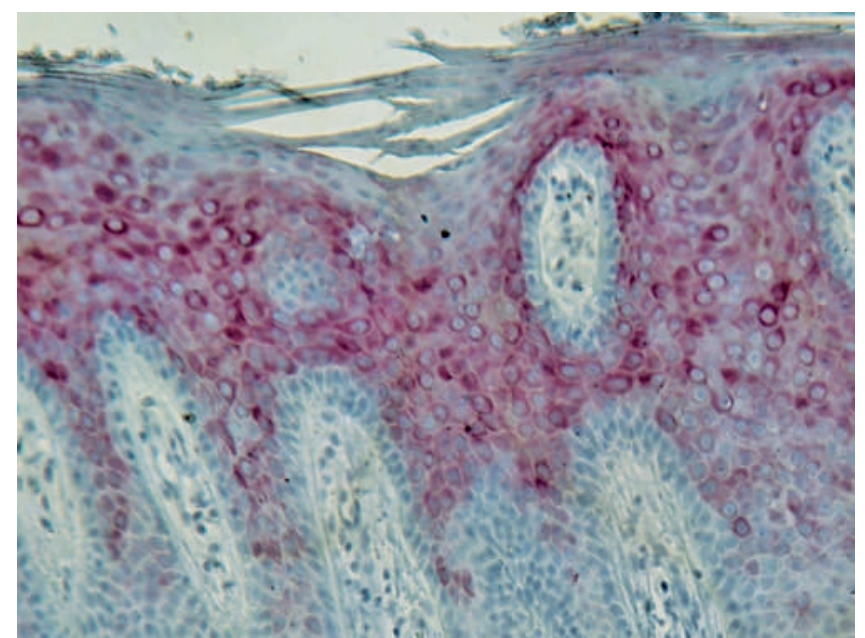

Abb. 5 Etanerceptpatient: CK16-Färbung: Befallene Haut vor Therapie (Vergrößerung 400fach).

\section{Ergebnisse}

$\nabla$

Beide Patientenkollektive weichen hinsichtlich des Krankheitsschweregrades vor Untersuchungsbeginn nicht merklich voneinander $a b$. Der PASI der Etanerceptgruppe beträgt vor Therapie $17,1(12,5 ; 24,4)$, in der Infliximabgruppe misst er $21,8(15,5$; 25,5).

Etanercept bewirkt eine statistisch auffällige Reduktion des PASI um 8,2 (14,7; 6,7). Nach Behandlung mit Etanercept ist der Juckreiz vermindert oder unverändert, die Epidermisdicke hat sich statistisch auffällig normalisiert. Der gleiche Trend zeichnet sich hinsichtlich der Proliferations- und Differenzierungsstörung ab. Die epidermalen und dermalen Entzündungsparameter (CD3, CD4, CD8 und CD45RO) haben sich insgesamt betrachtet jedoch nicht verringert ( $\bullet$ Tab. 1, $\bullet$ Abb. 2 u. 3-6).

Infliximab bewirkt einen statistisch auffälligen Abfall des PASI um 18,5 (23,6; 12,9). Auch Juckreiz und Epidermisdicke haben sich nach Therapie statistisch auffällig verbessert. Die Proliferations- und Differenzierungsstörung der Keratinozyten normalisiert sich ebenfalls (für Ki-67 und Involucrin statistisch auffällig). Im Gegensatz zur Etanerceptgruppe spricht in der Infliximabgruppe auch das entzündliche Infiltrat für eine Abheilung der psoriatischen Plaque. Die Verringerung der Memory-T-Zel-

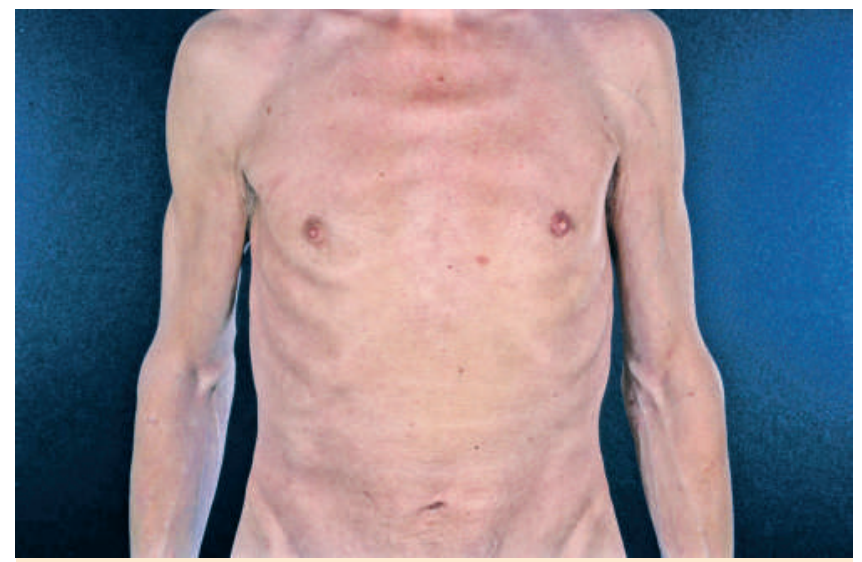

Abb. 4 Etanerceptpatient nach Therapie.

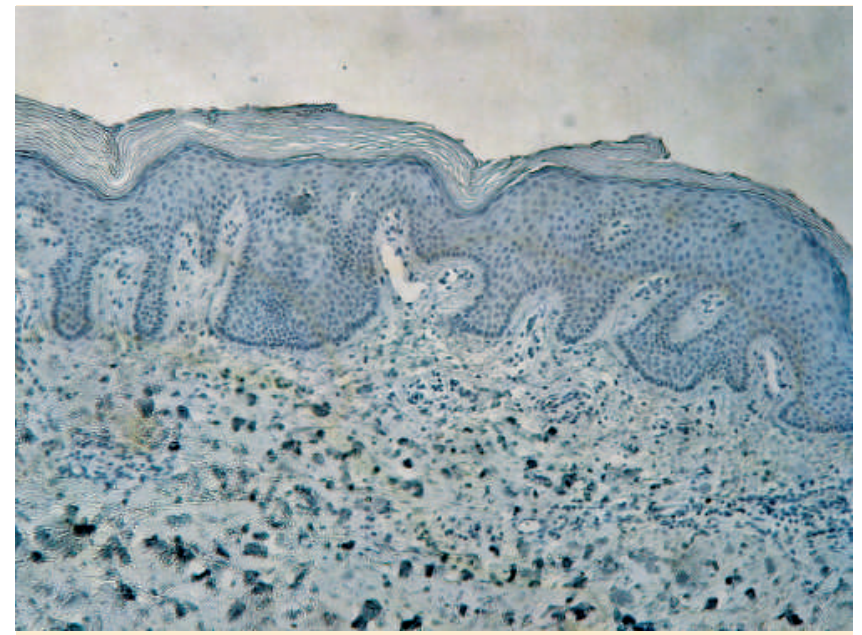

Abb. 6 Etanerceptpatient: CK16-Färbung: Ehemals befallene Haut nach Therapie (Vergrößerung 400fach). 


\begin{tabular}{|llll|}
\hline & Median $(\mathbf{Q 1} \mathbf{Q} \mathbf{Q})$ & $\begin{array}{l}\text { Minimum; } \\
\text { Maximum }\end{array}$ & $\begin{array}{l}\text { P-Wert } \\
\text { Vorzeichentest }\end{array}$ \\
\hline CK16 [mm] & $-0,09(-0,21 ; 0,03)$ & $-0,27 ; 0,10$ & 0,375 \\
\hline Filaggrin Mittelwert & $1(-5 ; 17)$ & $-11 ; 46$ & 1,000 \\
\hline Filaggrin Stratum corneum & $1(-0 ; 10)$ & $-2 ; 25$ & 0,625 \\
\hline Filaggrin Stratum granulosum & $0(-21 ; 30)$ & $-25 ; 90$ & 1,000 \\
\hline Involucrin & $-0,06(-0,15 ;-0,01)$ & $-0,20 ; 0,00$ & 0,063 \\
\hline Ki-67 [1/0,5mm] & $-33(-114 ; 61)$ & $-141 ; 83$ & 1,000 \\
\hline CD3 & $0,5(0,0 ; 2,3)$ & 0,$0 ; 3$, & 0,250 \\
\hline CD4 & $0,5(-1,5 ; 1,0)$ & $-3,0 ; 1,0$ & 1,000 \\
\hline CD8 & $0,0(-0,3 ; 0,3)$ & $-1,0 ; 1,0$ & 1,000 \\
\hline CD45RO & $-0,5(-1,5 ; 0,3)$ & $-3,0 ; 1$, & 0,625 \\
\hline Epidermisdicke $[\mathrm{mm}]$ & $-0,12(-0,18 ;-0,03)$ & $-0,19 ;-0,01$ & ${ }^{*} 0,031$ \\
\hline PASI & $-8,2(-14,7 ;-6,7)$ & $-29,0 ;-6,5$ & ${ }^{*} 0,031$ \\
\hline Juckreiz & $0,0(-1,5 ; 0,0)$ & $-3,0 ; 0,0$ & 0,500 \\
\hline
\end{tabular}

${ }^{*} \mathrm{p} \leq 0,05$ : statistisch auffällig.

\begin{tabular}{|llll|} 
& Median $(\mathbf{Q 1} \mathbf{Q} \mathbf{Q})$ & $\begin{array}{l}\text { Minimum; } \\
\text { Maximum }\end{array}$ & $\begin{array}{l}\text { p-Wert } \\
\text { Vorzeichentest }\end{array}$ \\
\hline CK16 [mm] & $-0,18(-0,20 ;-0,12)$ & $-0,23 ; 0,00$ & 0,063 \\
\hline Filaggrin Mittelwert & $60(15 ; 91)$ & $-1 ; 97$ & 0,219 \\
\hline Filaggrin Stratum corneum & $51(-7 ; 99)$ & $-29 ; 99$ & 0,375 \\
\hline Filaggrin Stratum granulosum & $75(45 ; 84)$ & $-2 ; 95$ & 0,219 \\
\hline Involucrin & $-0,24(-0,33 ;-0,15)$ & $-0,34 ;-0,05$ & ${ }^{*} 0,031$ \\
\hline Ki-67 [1/0,5mm] & $-106(-121 ;-47)$ & $-122 ;-7$ & ${ }^{*} 0,031$ \\
\hline CD3 & $0,0(-3,3 ; 0,0)$ & $-4,0 ; 0,0$ & 0,500 \\
\hline CD4 & $-1,5(-2,3 ;-0,8)$ & $-3,0 ; 0,0$ & 0,063 \\
\hline CD8 & $-1,5(-4,0 ; 0,3)$ & $-4,0 ; 1,0$ & 0,625 \\
\hline CD45RO & $-1,0(-2,0 ;-1,0)$ & $-2,0 ;-1,0$ & ${ }^{*} 0,031$ \\
\hline Epidermisdicke $[\mathrm{mm}]$ & $-0,20(-0,25 ;-0,12)$ & $-0,26 ;-0,03$ & ${ }^{*} 0,031$ \\
\hline PASI & $-18,5(-23,6 ;-12,9)$ & $-27,8 ;-11,9$ & ${ }^{*} 0,031$ \\
\hline Juckreiz & $-1,5(-2,3 ;-1,0)$ & $-3,0 ;-1,0$ & ${ }^{*} 0,031$ \\
\hline
\end{tabular}

Tab. 2 Infliximab: Intraindividuelle Differenzen der Werte vor und nach Therapie. Ein negatives Vorzeichen bedeutet einen Abfall der Werte nach Therapie und somit eine Verbesserung des pathologischen Hautbefundes und umgekehrt (außer bei Filaggrin, hier umgekehrt)

${ }^{*} p \leq 0,05$ : statistisch auffällig.

len (CD45RO+) hebt sich statistisch auffällig hervor $(p=0,031$, siehe $\odot$ Tab. 2, $\odot$ Abb. 2 u. 7-10).

Die Infliximabgruppe zeichnet sich bei allen klinischen und histologischen Befunden durch eine stärkere Verbesserung des Hautstatus aus. Hinsichtlich der Entzündungsreaktion (CD3+-TZellen) handelt es sich teilweise um einen statistisch auffälligen Unterschied ( $p=0,032$, 0 Tab. 1 u. 2). Die Abweichung zwischen beiden Medikamentengruppen manifestiert sich statistisch grenzwertig für PASI $(p=0,055)$, Juckreiz ( $p=0,067)$, Epidermisdicke $(p=0,077)$ und teilweise für den Differenzierungsgang der Keratinozyten (Involucrin $(p=0,054)$, Filaggrin Mittelwert $(p=0,055)$ und Stratum granulosum $(p=0,078))$. Bei beiden Therapien sind keine unerwünschten Wirkungen aufgetreten.

\section{Diskussion}

$\nabla$

Die in dieser Untersuchung gezeigte stärkere Wirksamkeit von Infliximab auf alle untersuchten Faktoren der Psoriasis, besonders bezüglich der T-Lymphozyten, kann auf verschiedenen Ursachen basieren. Ein Grund könnte das unterschiedliche, jeweils charakteristische Bindungsverhalten von Infliximab und Etanercept an TNF- $\alpha$ sein [23]. Infliximab bindet sowohl Monomere als auch Trimere des löslichen TNF- $\alpha$, während Etanercept auf die trimere Form beschränkt ist. Man nimmt an, dass Infliximab zusätzlich die Ausbildung des biologisch aktiven TNF- $\alpha$-Trimers verzögern oder verhindern kann. Etanercept besetzt nur zwei der drei Rezeptorbindungsstellen eines TNF- $\alpha$-Trimers, während
Infliximab alle blockiert $[23,24]$. Außerdem treten im Vergleich zu Etanercept mehr Infliximabmoleküle mit einer höheren Affinität an membrangebundenes TNF- $\alpha$ heran. Der Komplex von TNF- $\alpha$ und Infliximab ist im Gegensatz zu Etanercept stabil, was eine Dissoziation des Medikamentes von dem Zytokin verhindert. Obwohl beide Medikamente die durch transmembranes TNF- $\alpha$ vermittelte Aktivierung von humanen Endothelzellen hemmen, erweist sich Infliximab als signifikant effektiver [23]. Es wird vermutet, dass Infliximab ein "switch off" von bereits durch TNF- $\alpha$ aktivierten Zellen ermöglicht [25,3]. Infliximab neutralisiert die gesamte Bioaktivität von TNF- $\alpha$ (s. u.). Eine TNF$\alpha$-Blockade führt zu einer verringerten Expression von Adhäsionsmolekülen und Chemokinen [2,1], sodass weniger proinflammatorische Zellen in die Haut migrieren können. Es erscheint plausibel, dass eine stärkere Blockierung des proinflammatorischen Zytokins mit einer umfangreicheren Verbesserung der kutanen Entzündung und der gesamten Symptomatik einhergeht. Darauf könnten die Unterschiede der Patientengruppen bezüglich der Reduktion der T-Zellen beruhen.

Die Ergebnisse vorheriger Studien sprechen zumindest theoretisch dafür, dass Infliximab die Anzahl TNF- $\alpha$-produzierender Zellen reduziert [26]. Es aktiviert in vitro die ADCC (antibodydependent cell-mediated cytotoxicity) und die CDC (complement-dependent cytotoxicity) bei Zellen mit membranständigem TNF- $\alpha[24,27]$. Infliximab kann außerdem bei T-Zellen Apoptose induzieren $[28,29]$. Goedkoop et al. erklären bei Infliximab die Verminderung der epidermalen T-Zellen zusätzlich durch Deaktivierung des Gefäßendothels und Hemmung der 


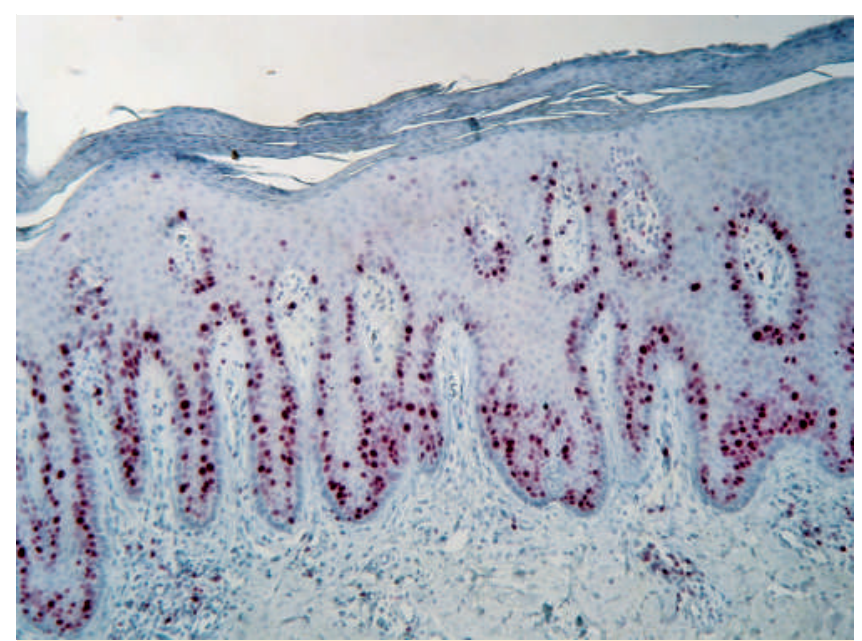

Abb. 7 Infliximabpatient: Ki-67-Färbung: Befallene Haut vor Therapie (Vergrößerung 400fach).

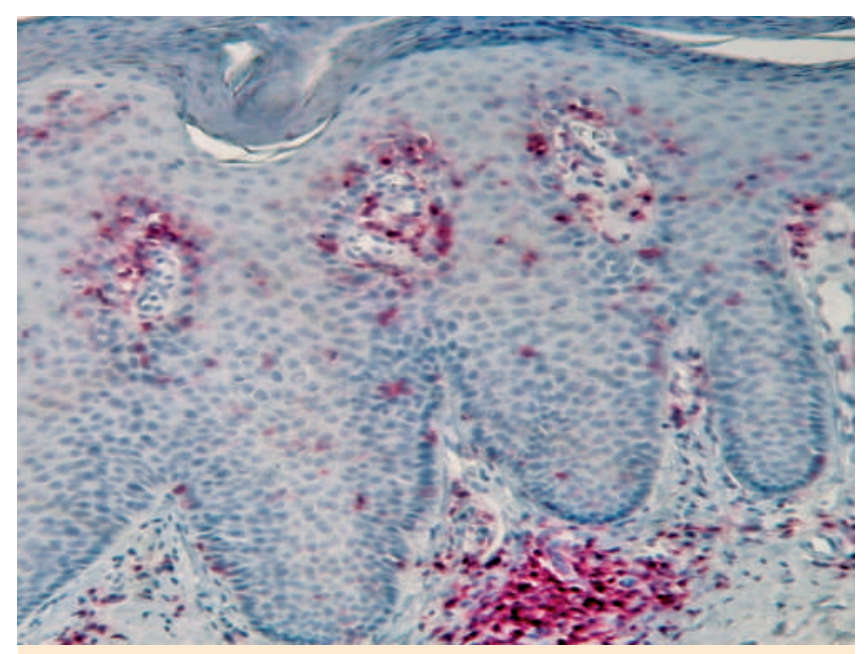

Abb.9 Infliximabpatient: CD45RO-Färbung: Befallene Haut vor Therapie (Vergrößerung 400fach).

Vaskularisation [30]. Im Gegensatz dazu wird bei Etanercept eine Hemmung des programmierten Zelltodes von T-Lymphozyten angenommen [17]. Trotz der Fc-Region vermittelt Etanercept keine CDC [8]. Diese Effekte könnten die gefundenen Unterschiede von Infliximab und Etanercept bezüglich des Lymphozyteninfiltrates nach Therapie erklären. Die vermutete Hemmung der Apoptose von T-Zellen durch Etanercept könnte insbesondere eine Ursache dafür sein, dass sich die kutanen T-Lymphozyten nach Etanercepttherapie nicht verringert haben.

Etanercept bindet sowohl TNF- $\alpha$ als auch Lymphotoxin [31]. Gottlieb et al. vermuten den Hauptwirkmechanismus der TNF$\alpha /$ Lymphotoxin-Blockade durch Etanercept in der supprimierten Expression von proinflammatorischen und proliferationsfördernden Genen, welche von TNF- $\alpha$ induziert werden, gefolgt von einer Verminderung der epidermalen dendritischen Zellen. Die zeitlich verzögerte Reduktion des T-Zellinfiltrates kann sekundär als Folge davon betrachtet werden [31]. Die Normalisierung des T-Zellinfiltrates nach Etanercepttherapie könnte folglich noch zu einem späteren Zeitpunkt erfolgen.

Zusätzlich zur Therapiedauer könnte die unterschiedliche Wirksamkeit von Etanercept und Infliximab auch auf den jeweiligen Dosierungen beruhen. Die Effektivität der Psoriasistherapie mit

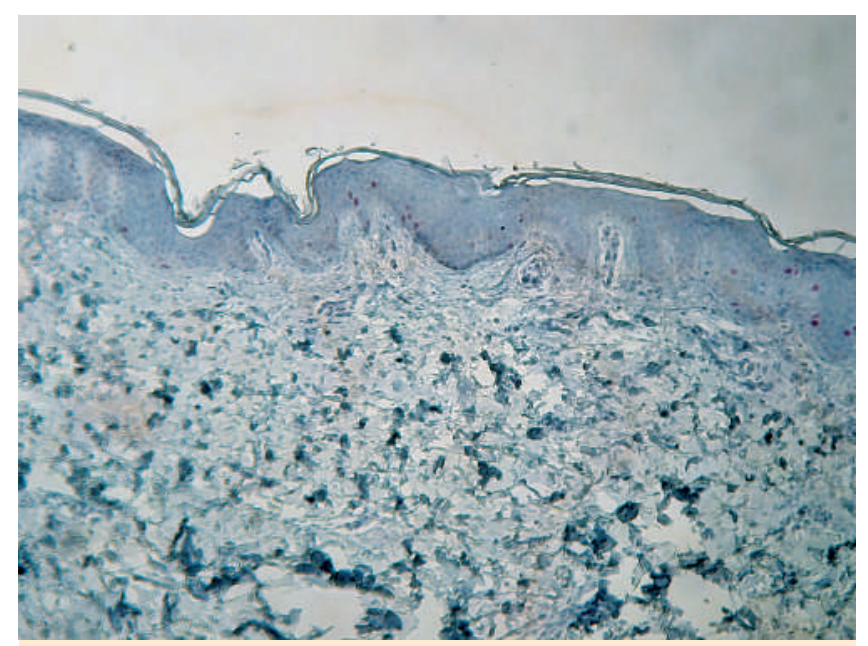

Abb. 8 Infliximabpatient: Ki-67-Färbung: Ehemals befallene Haut nach Therapie (Vergrößerung 400fach).

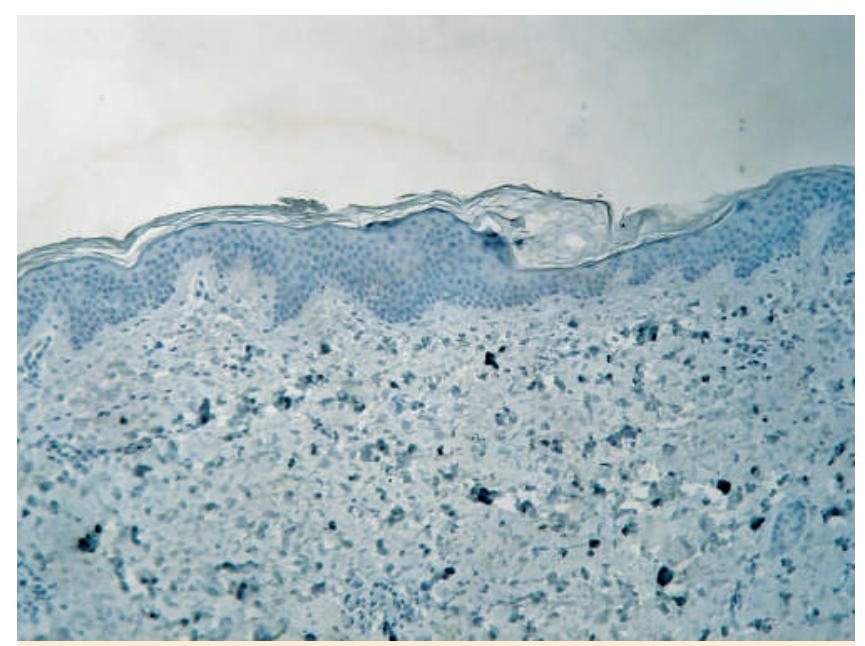

Abb. 10 Infliximabpatient: CD45RO-Färbung: Ehemals befallene Haut nach Therapie (Vergrößerung 400fach).

Etanercept steigt nämlich nicht nur mit zunehmender Behandlungsdauer (24 Wochen vs. 12 Wochen) [6], sondern auch mit zunehmender Dosis $(50 \mathrm{mg} / \mathrm{kg}, 2$-mal wöchentlich s.c. vs. $25 \mathrm{mg} / \mathrm{kg}$, 2-mal wöchentlich s.c.) [32]. Bei Infliximab wird im Gegensatz dazu nach drei Infusionen in Woche 0, 2 und 6 die maximale Wirkung in Woche 10 erzielt, ab Woche 14 steigt der PASI wieder an [33].

Unter Berücksichtigung der vielfältigen Funktionen von TNF- $\alpha$ bezüglich der Granulombildung erscheint es plausibel, dass die Blockade von TNF- $\alpha$ das Risiko von Infektionen, die normalerweise durch Granulombildung beherrscht werden (z. B. Tbc), erhöht [34]. Im Gegensatz zur Etanercepttherapie wirkt die Infliximabtherapie bei M. Crohn und führt signifikant schneller und öfter zu etwaigen Nebenwirkungen (siehe Einleitung) [14-17]. Wallis und Ehlers [34] stellen in diesem Zusammenhang drei Hypothesen auf:

- Unterschiedliche Hemmung der Signalübertragung und die Folgen

Infliximab blockiert die Signaltransduktion über beide TNF$\alpha$-Rezeptoren (p55 und p75), während Etanercept den Weg über TNFRp75 zumindest teilweise offen lässt [23,4]. Studien zeigen, dass die Signalübertragung über TNFRp55 meist mit 
schädlichen, proinflammatorischen Ereignissen einhergeht, während die Signalübertragung über TNFRp75 immunmodulatorische, krankheitsverbessernde Funktionen unterstützt [35].

- Unterschiedlich starke Hemmung der Bioverfügbarkeit von TNF

Im Gegensatz zu Etanercept bindet Infliximab TNF- $\alpha$ schnell und irreversibel [23]. Bei hohen Spiegeln neutralisiert Infliximab die gesamte Bioaktivität, während Etanercept bioverfügbares TNF- $\alpha$ von der Produktionsstelle zu Orten mit geringerer Konzentration umverteilt. Es gibt möglicherweise ein Fenster, in welchem die TNF- $\alpha$-Spiegel hoch genug sind, um die Unterhaltung der Granulome zu unterstützen, aber niedrig genug, um die Aktivität von manchen chronischen inflammatorischen Krankheiten wie z.B. M. Crohn zu reduzieren [34].

- Unterschiede bezüglich der Induktion von Apoptose Infliximab löst bei Monozyten von M. Crohn-Patienten Apoptose aus [36]. Dieser Prozess ist defizient bei Patienten mit aktivem M. Crohn [37]. Falls Infliximab gegen mycobakterielle Antigene gerichtete T-Zellen durch die Auslösung von Apoptose eliminiert, würde das die Reaktivierung von Tbc erklären. Der programmierte Zelltod ist ein anerkannter Mechanismus für den Rückgang der T-Zellen bei Tbc und HIV. Die Induktion von ADCC und CDC durch Infliximab (s.o.) könnte den relativ hohen Anteil der disseminiert auftretenden Tbc bei Infliximabtherapie erklären, da die lysierten Makrophagen des Granuloms die Mycobakterien in den Blutstrom ausschütten könnten [34].

Obwohl Etanercept und Infliximab ein gemeinsames Ziel haben, unterscheiden sie sich im Wirkungs- und Nebenwirkungsprofil. Die Ergebnisse von klinischen und experimentellen Studien können zur Schaffung einer neuen Generation von TNF- $\alpha$-Blockern führen, bei denen der therapeutische Erfolg maximiert, die Nebenwirkungen jedoch minimiert werden [34]. Aufgrund der schnellen effektiven Wirkung und guten Verträglichkeit stellen die TNF- $\alpha$-Blocker eine Alternative zur herkömmlichen Therapie der Psoriasis dar.

\section{Danksagung}

Wir danken dem Institut für Medizinische Biometrie, Epidemiologie und Informatik der Johannes Gutenberg-Universität Mainz für die Zusammenarbeit bezüglich der statistischen Auswertung.

\section{Abstract}

\section{Anti-TNF- $\alpha$-Therapy of Psoriasis with Infliximab or Etanercept. Clinical, Histological and Immunohisto- chemical Course}

TNF- $\alpha$ plays a central role in the pathogenesis of psoriasis. Recently, it has been demonstrated that psoriatic skin lesions showed improvement during anti-TNF- $\alpha$-therapy with etanercept or infliximab. These agents act in a different, not yet completely understood manner. The purpose of this study was to assess the effects of short-term therapy by treating 6 patients with etanercept and another 6 patients with infliximab. In each case, the pathological, histological and clinical signs of psoriasis vul- garis were analysed and compared. PASI, pruritus, epidermal thickness, markers of cutaneous inflammation, keratinocyte proliferation and differentiation were evaluated. Treatment with etanercept: PASI was diminished by 8.2 (14.7; 6.7; $\mathrm{p}=0.031)$. Pruritus after etanercept treatment remained either unchanged or was reduced. Epidermal thickness was normalized $(p=0.031)$. The same tendency was observed in markers of keratinocyte proliferation and differentiation, but, in general, the number of epidermal and dermal inflammatory cells did not decrease. Treatment with infliximab: PASI was diminished by 18.5 (23.6; $12.9 ; p=0.031)$. Pruritus and epidermal thickness were diminished $(p=0.031)$. Markers of keratinocyte proliferation and differentiation were reduced (Ki-67 and CD45RO+ significantly, $\mathrm{p}=0.031$ ). In contrast to the etanercept treatment T-cells in skin were also reduced (CD45RO+-cells, $\mathrm{p}=0.031$ ). The patients who received infliximab showed greater improvement in all analysed clinical and histological symptoms of psoriasis. Regarding the cutaneous T-cells infiltrate, a reduction of CD3+-T-cells $(p=0.032$ ) occurred. The results of this study correspond to the different mechanisms of action of infliximab and etanercept and outline the key position of TNF- $\alpha$ for psoriasis.

\section{Literatur}

1 Winterfield LS, Menter A. Infliximab. Dermatol Ther 2004; 17 (5): 409 426

2 Ettehadi P, Greaves MW, Wallach D, Aderka D, Camp RD. Elevated tumour necrosis factor-alpha (TNF-alpha) biological activity in psoriatic skin lesions. Clin Exp Immunol 1994; 96 (1): 146 - 151

3 Gottlieb AB. Infliximab for psoriasis. J Am Acad Dermatol 2003; 49 (2 Suppl): S112-117

4 Grell M, Douni E, Wajant $H$ et al. The transmembrane form of tumor necrosis factor is the prime activating ligand of the $80 \mathrm{kDa}$ tumor necrosis factor receptor. Cell 1995; 83 (5): 793-802

5 Aderka D, Engelmann H, Maor Y, Brakebusch C, Wallach D. Stabilization of the bioactivity of tumor necrosis factor by its soluble receptors. J Exp Med 1992; 175 (2): $323-329$

6 Gottlieb AB, Chaudhari U, Mulcahy LD, Li S, Dooley LT, Baker DG. Infliximab monotherapy provides rapid and sustained benefit for plaquetype psoriasis. J Am Acad Dermatol 2003; 48 (6): 829-835

7 Schopf RE, Aust H, Knop J. Treatment of psoriasis with the chimeric monoclonal antibody against tumor necrosis factor alpha, infliximab. J Am Acad Dermatol 2002; 46 (6): 886 - 891

8 Goffe B, Cather JC. Etanercept: An overview. J Am Acad Dermatol, 2003; 49 (2 Suppl): S105 - 111

9 Mohler KM, Torrance DS, Smith CA et al. Soluble tumor necrosis factor (TNF) receptors are effective therapeutic agents in lethal endotoxemia and function simultaneously as both TNF carriers and TNF antagonists. J Immunol 1993; 151 (3): 1548 - 1561

10 Peppel K, Crawford D, Beutler B. A tumor necrosis factor (TNF) receptorIgG heavy chain chimeric protein as a bivalent antagonist of TNF activity. J Exp Med 1991; 174 (6): 1483-1489

11 Knight DM, Trinh H, Le J et al. Construction and initial characterization of a mouse-human chimeric anti-TNF antibody. Mol Immunol 1993; 30 (16): $1443-1453$

12 Targan SR, Hanauer SB, van Deventer SJ et al. A short-term study of chimeric monoclonal antibody cA2 to tumor necrosis factor alpha for Crohn's disease. Crohn's Disease cA2 Study Group. N Engl J Med 1997; 337 (15): 1029- 1035

13 Sandborn WJ, Hanauer SB, Katz $S$ et al. Etanercept for active Crohn's disease: a randomized, double-blind, placebo-controlled trial. Gastroenterology 2001; 121 (5): 1088-1094

14 Fleischmann $R$. Safety and efficacy of disease-modifying antirheumatic agents in rheumatoid arthritis and juvenile rheumatoid arthritis. Expert Opin Drug Saf 2003; 2 (4): 347-365

15 Fleischmann R, Iqbal I, Nandeshwar P, Quiceno A. Safety and efficacy of disease-modifying anti-rheumatic agents: focus on the benefits and risks of etanercept. Drug Saf 2002; 25 (3): 173-197

16 Gardam MA, Keystone EC, Menzies R, Manners S, Skamene E, Long R, Vinh $D C$. Anti-tumour necrosis factor agents and tuberculosis risk: 
mechanisms of action and clinical management. Lancet Infect Dis 2003; 3 (3): $148-155$

17 Wallis RS, Kyambadde P, Johnson JL et al. A study of the safety, immunology, virology, and microbiology of adjunctive etanercept in HIV1-associated tuberculosis. AIDS 2004; 18 (2): 257-264

18 Fredriksson $T$, Pettersson U. Severe psoriasis-oral therapy with a new retinoid. Dermatologica 1978; 157 (4): 238-244

19 Castelijns FA, Gerritsen MJ, van Vlijmen-Willems IM, van Erp PE, van de Kerkhof PC. The epidermal phenotype during initiation of the psoriatic lesion in the symptomless margin of relapsing psoriasis. J Am Acad Dermatol 1999; 40 (6 Pt 1): 901 - 909

20 Gerritsen MJ, Rulo HF, Arnold WP, de Kerkhof PCV. Response of the clinically uninvolved skin of psoriatic patients to repeated tape stripping during cyclosporin A treatment. Br J Dermatol 1994; 130 (2): 181 - 188

21 Gerritsen MJ, van Pelt JP, van de Kerkhof PC. Response of the clinically uninvolved skin of psoriatic patients to tape stripping during acitretin treatment. Acta Derm Venereol 1996; 76 (1): 6 - 9

22 Fritsch P. Dermatologie, Venerologie. (Kapitel 13.1.1 Psoriasis vulgaris). Berlin: Springer, 2004: 360-374

23 Scallon B, Cai A, Solowski N, Rosenberg A, Song XY, Shealy D, Wagner C. Binding and functional comparisons of two types of tumor necrosis factor antagonists. J Pharmacol Exp Ther 2002; 301 (2): 418 - 426

24 Scallon BJ, Moore MA, Trinh H, Knight DM, Ghrayeb J. Chimeric antiTNF-alpha monoclonal antibody cA2 binds recombinant transmembrane TNF-alpha and activates immune effector functions. Cytokine 1995; 7 (3): $251-259$

25 Chaudhari U, Romano P, Mulcahy LD, Dooley LT, Baker DG, Gottlieb AB. Efficacy and safety of infliximab monotherapy for plaque-type psoriasis: a randomised trial. Lancet 2001; 357 (9271): $1842-1847$

26 Maini RN, Feldmann M. How does infliximab work in rheumatoid arthritis? Arthritis Res 2002; 4 Suppl 2: S22 - 28

27 Kohno $T$ et al. Differences in Fc Receptor and C1q Binding in Tumor Necrosis Factor (TNF) Antagonists may Contribute to Differences in Mechanisms of Action. Posterbeitrag (Poster 666) präsentiert auf 66th Annual Meeting Society for Inverstigative Dermatology, St. Louis, MO, USA, 4. - 7. Mai 2005, amgen Inc. Thousand Oaks, CA
28 den Brande JMHV, Braat $H$, van den Brink GR et al. Infliximab but not etanercept induces apoptosis in lamina propria T-lymphocytes from patients with Crohn's disease. Gastroenterology 2003; 124 (7): 1774 1785

29 ten Hove T, van Montfrans C, Peppelenbosch MP, van Deventer SJH. Infliximab treatment induces apoptosis of lamina propria $\mathrm{T}$ lymphocytes in Crohn's disease. Gut 2002; 50(2): 206-211

30 Goedkoop AY, Kraan MC, Teunissen MBM et al. Early effects of tumour necrosis factor alpha blockade on skin and synovial tissue in patients with active psoriasis and psoriatic arthritis. Ann Rheum Dis 2004; 63 (7): $769-773$

31 Gottlieb AB, Chamian F, Masud S et al. TNF Inhibition Rapidly DownRegulates Multiple Proinflammatory Pathways in Psoriasis Plaques. J Immunol 2005; 175 (4): 2721 - 2729

32 Leonardi CL. Efalizumab: an overview. J Am Acad Dermatol 2003; 49 (2 Suppl): S98 - 104

33 Gottlieb $A B$, Evans $R$, Li $S$ et al. Infliximab induction therapy for patients with severe plaque-type psoriasis: a randomized, double-blind, placebo-controlled trial. J Am Acad Dermatol 2004; 51 (4): 534 - 542

34 Wallis RS, Ehlers $S$. Tumor necrosis factor and granuloma biology: explaining the differential infection risk of etanercept and infliximab. Semin Arthritis Rheum 2005; 34 (5 Suppl1): 34-38

35 Kassiotis G, Kollias G. Uncoupling the proinflammatory from the immunosuppressive properties of tumor necrosis factor (TNF) at the p55 TNF receptor level: implications for pathogenesis and therapy of autoimmune demyelination. J Exp Med 2001; 193 (4): 427-434

36 Lügering A, Schmidt M, Lügering N, Pauels HG, Domschke W, Kucharzik $T$. Infliximab induces apoptosis in monocytes from patients with chronic active Crohn's disease by using a caspase-dependent pathway. Gastroenterology 2001; 121 (5): 1145 - 1157

37 Boirivant M, Marini M, Felice GD, Pronio AM, Montesani C, Tersigni R, Strober W. Lamina propria T cells in Crohn's disease and other gastrointestinal inflammation show defective CD2 pathway-induced apoptosis. Gastroenterology 1999; 116 (3): 557-565 